EPJ Web of Conferences 108, 02036 (2016)

DOI: $10.1051 /$ epjconf/201610802036

C Owned by the authors, published by EDP Sciences, 2016

\title{
Prediction of Liquid Sodium Flow Rate through the Core of the IBR-2M Reactor Using Nonlinear Autoregressive Neural Networks
}

\author{
G. Ososkov ${ }^{1, a}$, Yu. Pepelyshev ${ }^{1, b}$, Ts. Tsogtsaikhan ${ }^{1,2, c}$ \\ ${ }^{1}$ Joint Institute for Nuclear Research, 141980 Dubna, Moscow region, Russia \\ ${ }^{2}$ Institute of physics and technology, MAS, Mongolia
}

\begin{abstract}
This paper presents an artificial neural network method for long-term prediction of liquid sodium flow rate through the core of the IBR-2M reactor. The nonlinear autoregressive neural network (NAR) with local feedback connection has been considered as the most appropriate tool for such a prediction. The predicted results were compared with experimental values. NAR model predicts slow changes of liquid sodium flow rate up to two days with an error less than 5\%.
\end{abstract}

\section{Introduction}

The IBR-2M pulsed reactor with periodic pulse operation (modernized version of IBR-2 reactor) was commissioned with the average power of $2 \mathrm{MW}$ at the Joint Institute for Nuclear Research (Dubna, Russia) in 2012. The reactor generates short $200 \mu$ s neutron pulses at half-width with period $0.2 \mathrm{~s}$ and amplitude of $1830 \mathrm{MW}$. The reactor coolant is liquid sodium. The variation in the temperature and the flow rate of the liquid sodium through the core affects the reactivity fluctuation and power. The goal of this work is to predict the liquid sodium flow rate through the core of the IBR-2M during reactor operation. It is important for ensuring safety and reliable operation of the reactor. Earlier, artificial neural network (ANN) was used widely to study the pulse energy noise dynamics of IBR-2 [1-4]. With the development of powerful computation systems and also such new methods as nonlinear autoregressive neural network (NAR) [6], their application for data analysis to forecasting of the liquid sodium flow rate on the modernized IBR-2M reactor is again of major importance.

\section{Experimental data and basics of choice modelling}

The original time series to be predicted (on the liquid sodium flow rate) were recorded within four days of reactor cycle. The measurement period (or sampling frequency) amounted to $0.1 \mathrm{~s}$. The reactor operates continuously for a 12 day cycle followed by a shut down to prepare for the next

\footnotetext{
a e-mail: ososkov@jinr.ru

be-mail: pepel@nf.jinr.ru

ce-mail: tsolmon@nf.jinr.ru
} 
experiments. The length of the time series during the measurement reached $\sim 5 \cdot 10^{7}$ values. Based on the connection pattern, the ANN can be grouped in two categories: feed forward networks, in which ANN graphs have no loops, and recurrent (or feedback) networks, in which loops occur because of feedback connection. Neurons of feed forward networks, called multilayer perceptrons, are organized into several layers that have unidirectional connection between them. The neural network technique was chosen as a predictor. Several types of feed forward multilayer perceptrons and the ARIMA model [5] were tested, but the best results were achieved only when the feedback was included into the neural network scheme. Generally, the feed forward networks are static, that is, they produce only one set of output values rather than a sequence of values from a given input. Feed-forward networks are memory-less in the sense that their response to an input is independent of the previous network state. Feedback networks, on the other hand, are dynamic systems. When a new input pattern is presented, the neuron outputs are computed. Because of the feedback paths, the inputs to each neuron are then modified, which leads the network to enter a new state [6]. Such types of neural networks are commonly used for modelling dynamic nonlinear time series. The nonlinear autoregressive neural network (NAR) is a recurrent dynamic network based on an autoregressive model with feedback connection [7-9].The NAR type of neural network with feedback connection is widely applied for noisy time series prediction. In particular, in this study it is applied to predict the liquid sodium flow rate through the core of the IBR-2M. NAR prediction results are more accurate as compared to the common feed forward networks.

\section{Nonlinear autoregressive neural network}

The artificial neural networks are one of the basic directions of the modern theory of the artificial intelligence [6]. As an intelligent system, an ANN has the ability to recognize and predict time series data and its nonlinear characteristics. The output of a neuron can be written parametrically as the following equation,

$$
y_{j}=f\left(b+\sum_{i} w_{i j} x_{i}\right)
$$

$b$ is the bias for neuron, $f$ is the activation function, $w_{i j}$ are the weights, $x_{i}$ is the input and $y$ represents the output $[6,8,10]$. In this work, the type of activation function $f$ was chosen as a sigmoid for hidden layer,

$$
f(x)=\frac{1}{1+e^{-\alpha x}},
$$

where $x$ is the argument of the function, $\alpha$ is a coefficient.

In the process of NAR training the network does not use the feedback connection. Two days of liquid sodium flow rate data are used as the training sample, which consists of 700 vectors with $n+1$ components $\{G(t-1), G(t-2), \ldots, G(t-n) ; \hat{G}(t)\}$, where the first $n$ components are for input and the last one for output, $G(t)$ denotes the liquid sodium flow rate through the core of the reactor; $\hat{G}(t)$ is the output (predicted value); $n$ is the feedback delay.

After training with all the weights fixed, the next predicting stage demands to include into the NAR structure the feedback connection, which brings dynamics to the NAR output. NAR uses the weight values obtained on the previous stage and additionally includes the feedback connection. The structure of the NAR NN is shown in figure 1.

The NARs are based on back-propagation training algorithms that were used for long-term time series prediction with MATLAB [10]. In our application NAR has $n$ inputs, one output neuron and a single hidden layer with 20 neurons. After the autocorrelation analysis of the liquid sodium flow 
rate variables, the number of feedback delays was set to $n=44$. The NAR structure with 20 hidden neurons was found to be the most effective after testing the efficiency of networks with 10 and 15 neurons. In the training phase, the Levenberg-Marquardt back-propagation algorithm [11] is used to minimize the network error. The data set of training was divided into three parts: $70 \%$ for training, $15 \%$ for validation, and $15 \%$ as test data. The training sample included the above mentioned 700 values from the first two days of liquid sodium flow rate data of reactor operation. The open loop network closed after training and the addition of the feedback connection. After training, the first two days of data are applied to predict values $\hat{G}(t)$ for the next two days. The predicted values are then compared with the experimental ones to validate the NAR efficiency.

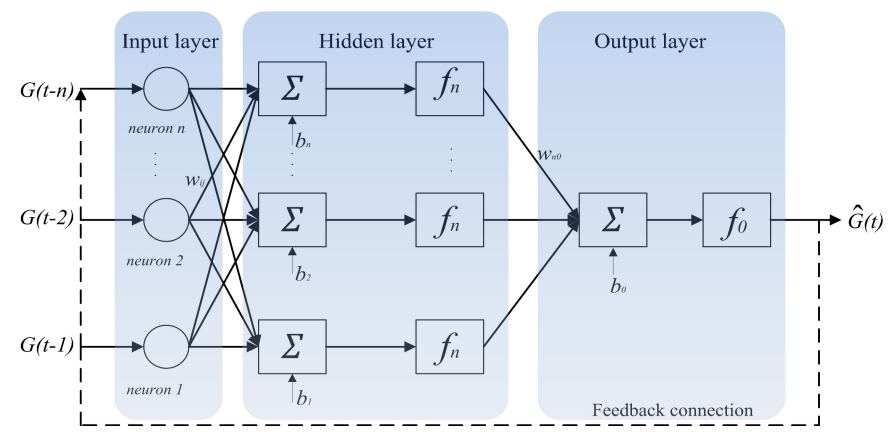

Figure 1. Structure of NAR network

\section{Results and discussion}
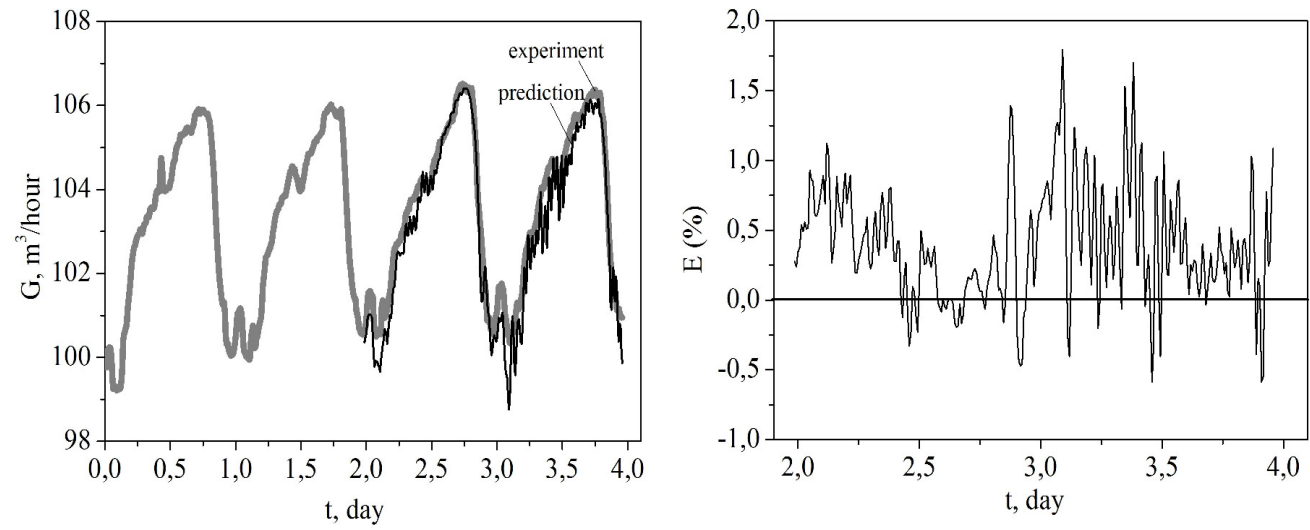

Figure 2. Comparison between measured daily liquid sodium flow rate through the core of the IBR-2M and predicted by the proposed NAR model (on the left). Prediction error (relative difference) between experimental and predicted data (on the right).

The goal was to predict two days data points $\hat{G}(t)$ from two previous days data $G(t)$. The results of the liquid sodium flow rate prediction and the prediction error are shown in figure 2 . Figure 3 

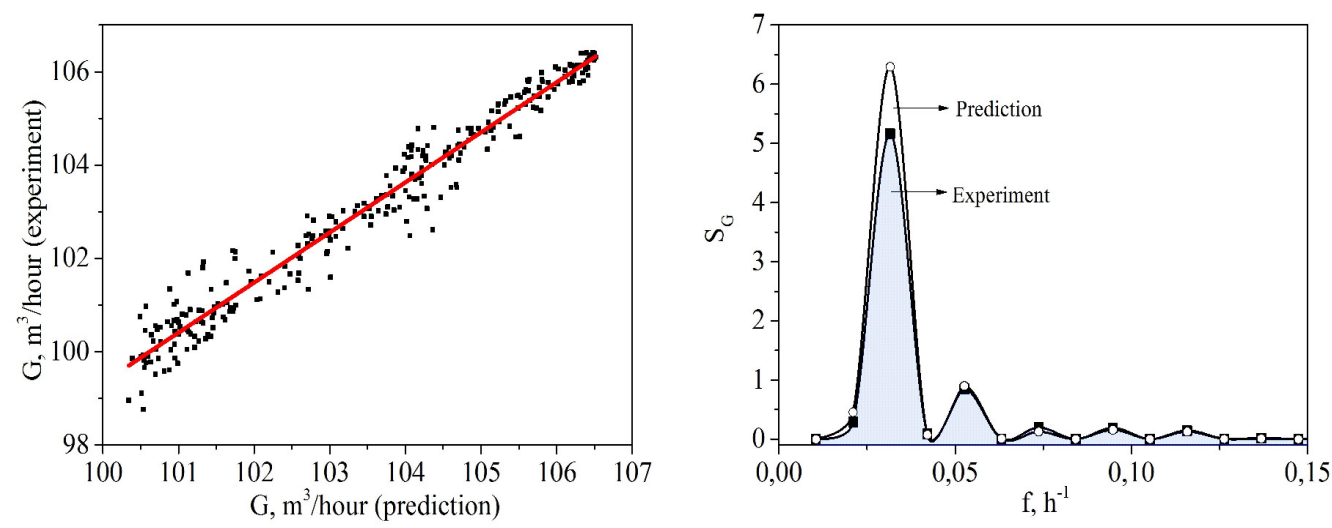

Figure 3. Almost linear relationship (on the left) and power spectrum (on the right) of experimental results and predicted data using the proposed NAR model

illustrates the comparison of experimental and predicted data in terms of the linear relationship and the power spectrum. There is very good agreement between them $(R=0.98)$. The spectral shape and power spectrum amplitude of the liquid sodium flow rate are practically the same.

\section{Conclusion}

In this paper, we presented a predicting methodology for liquid sodium flow rate through the core of the IBR-2M based on the nonlinear autoregressive neural network.

The study was fulfilled to choose the best predictor for the liquid sodium flow rate through the core of the IBR-2M during a reactor operation. Such standard methods, as the conventional feed forward multilayer perceptrons and the ARIMA model were tested, but they were unable to realize even one day of satisfactory prediction. The nonlinear autoregressive neural network (NAR) model allows to predict slow changes of the liquid sodium flow rate up to two days within a prediction error less than $5 \%$. The very good agreement $(R=0.98)$ was demonstrated between experimental results and data predicted by the proposed NAR model.

Thus the results produced by the NAR network are more accurate with better long-term predicting than the standard methods.

\section{References}

[1] Pepelyshev Yu.N. and Dzwinel W., Progress in Nuclear Energy 43, No. 1-4, 145-150 (2003)

[2] Dzwinel W., Pepyolyshev Yu.N. and Dlugopolski J., Proc. of International Seminar - IV Kolloquium fur Maschinen Diagnostik, 14-15 Marzec 1996, Technical University in Dresden, Germany, 571-580 (1996)

[3] Dzwinel W. and Pepyolyshev Yu.N., Proceedings of the SMORN VII International Symposium on Nuclear Reactor Surveillance and Diagnostics, Avignon, 19-23 June 1995, France, 1, 4.5, 8 pp. 
[4] Dlugopolski J., Dzwinel W. and Pepyolyshev Yu. N., Proc. of the 10-th Summer School on Computing Techniques in Physics, High Performance Computing in Science, 5-14 September 1995, Skalsky Dvur, Czech Republic

[5] Mills T., The Econometric Modelling of Financial Time Series. 2nd ed. Cambridge University Press: Cambridge 1999

[6] Haykin S., Neural networks: a comprehensive foundation. 2nd ed. Prentice Hall, 1998

[7] Giles L.C., Lawrence S. and Tsoi A.C., Machine Learning 44, 161-183 (2001)

[8] Akhterov A.V. and Kiril'chenko A.A., Bases of the Theoretical Robotechnics. Artificial Neural Networks. (Review) (Inst. Math., the Russian Academy of Science, Moscow, 2008)

[9] Huang J., Korolkiewicz M., Agrawal M. and Boland J., Sol. Energy 87, 136-149 (2013)

[10] Demuth H., Beale M. and Hagan M., Neural Network Toolbox Revised for Version 6.0.3 (September 2009)

[11] H. P. Gavin., http://people.duke.edu/ hpgavin/ce281/lm.pdf 
